\title{
PRELIMINARY STUDY ON THE FRAGILITY CURVES FOR HIGHWAY BRIDGES IN TAIWAN
}

\author{
Wen-I Liao* and Ching-Hsiung Loh
}

\begin{abstract}
This paper describes procedures of bridge fragility analysis for the highway bridges on the south-north freeway in Taiwan, and studies the evaluation of parameters used in bridge damage assessment. The fragility curves are used to represent the probabilities that structural damage, under various levels of seismic excitation, exceed specified damage states. Since it is neither necessary nor practical to evaluate individual bridges, bridge classification and mapping scheme plays an important role. Calculation of site-specific seismic demand and damage functions (i.e., capacity curves and fragility curves) are the key features in bridge damage assessment and earthquake loss estimation.
\end{abstract}

Key Words: fragility analysis, capacity curve, highway bridge.

\section{INTRODUCTION}

Earthquake loss estimation methodology, integrated with the geographic information system (GIS) and designed to run on personal computers, has been developed in the United States and is referred to as HAZUS (RMS, 1999). Essentially, Haz-Taiwan follows the approach used in HAZUS. To accommodate special environmental and engineering practices in Taiwan, minor modifications in analysis models and parameters have been created by Yeh et al., (1999). As shown in Fig. 1, the methodological framework is mainly composed of input databases, analysis modules and application software. The input databases consist of inventory data from GIS information, earthquake hazard and geologic data maps, and analysis parameters. The analysis modules take the required inventory data and analysis parameters as inputs, conduct risk assessment and loss estimation for earthquake scenarios based on site-specific earthquake hazard outputs, and output estimated

*Corresponding author. (Tel: 886-7-5919222; Fax: 886-75919376; Email: wiliao@nuk.edu.tw)

W. I. Liao is with the Department of Civil and Environmental Engineering, National University of Kaohsiung, Kaohsiung, Taiwan 811, R.O.C.

C. H. Loh is with the Department of Civil Engineering, National Taiwan University, and National Center for Research on Earthquake Engineering, Taipei, Taiwan 106, R.O.C. data in the resulting databases. When integrated with commercial GIS software, this PC-based application software is used to execute user's requests, to display input/output databases in both tabular and graphical forms, to generate summary reports, etc.

The Chi-Chi earthquake caused significant losses to civil infrastructure. The need to mitigate seismic risk to civil infrastructure has become evident after this earthquake. Bridge damage assessment is one of the important issues in earthquake loss estimation. Assessment of damage to highway systems from earthquake and estimation of consequent loss provide valuable information for post-earthquake planning and risk mitigation. The methodology can serve as a tool in the decision process for emergency response operations and retrofitting of critical structures in the system as a means of pre-disaster mitigation. The vulnerability assessment of bridges includes hazard analysis, classification of the critical components of bridge structures and fragility analysis. To evaluate the seismic response of a number of bridges, it is neither necessary nor practical to assess individual bridge damage. Bridges first can be categorized into different classes according to their structural characteristics. Since the structural system is the major factor in assessing structural performance, loss of function and casualties, damage is predicted based on the bridge model type. One of the most important issues in evaluating the seismic 
Table 1 Haz-Taiwan bridge classification for South-North highway

\begin{tabular}{lcccc}
\hline No. of span & Superstructure & Pier type & Design & Class \\
\hline Single span & & & Conventional & TYPE 1C \\
& & & Seismic & TYPE 1S \\
\hline \multirow{4}{*}{ Simply supported } & Single column & Conventional & TYPE 2C \\
& & pier & Seismic & TYPE 2S \\
\cline { 3 - 4 } & & Multiple column & Conventional & TYPE 3C \\
& & bent & Seismic & TYPE 3S \\
\cline { 2 - 4 } Multiple span & Pier wall & Conventional & TYPE 4C \\
& & & Seismic & TYPE 4S \\
\cline { 2 - 4 } & & Single column & Conventional & TYPE 5C \\
& & pier & Seismic & TYPE 5S \\
\cline { 2 - 4 } & Continuous & Multiple column & Conventional & TYPE 6C \\
& & bent & Seismic & TYPE 6S \\
\cline { 2 - 4 } & & Pier wall & Conventional & TYPE 7C \\
& & & Seismic & TYPE 7S \\
\cline { 2 - 4 } & Others & & Conventional & TYPE 8C \\
& & & Seismic & TYPE 8S \\
\hline
\end{tabular}

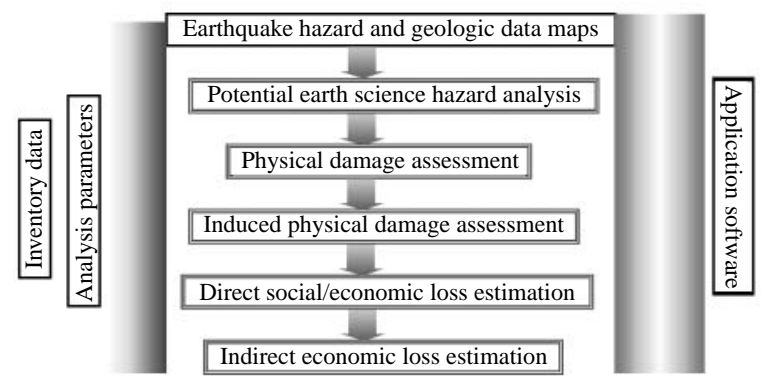

Fig. 1 Framework of methodology in Haz-Taiwan

damage to structures is the so-called fragility curve. The fragility curves are used to represent the probabilities that the structural damages, under various levels of seismic excitation, exceed specified damage states. Each point on the fragility curve represents the probability that the spectral response under a certain level of ground shaking, is greater than the response associated with a certain damage state.

This paper presents the methods developed to enhance the transportation lifelines module in HAZTaiwan for highway bridges. The objective of this paper is to provide the bridge classifications and fragility functions on the South-North Freeway in Taiwan by utilizing the available investigation data. The organization of this paper is as follows. First, the available bridge data from the South-North freeway in Taiwan are reviewed and a classification based on the available data to be implemented in Haz-Taiwan is proposed. Second, the description of failure mechanisms and criteria in different damage states adopted in this research are summarized. Third, an analytic methodology for fragility analysis for bridges and examples for freeway bridges are presented. Parameters for describing the fragility curves for each class are also generated and shown in Table.

\section{BRIDGE CLASSIFICATIONS}

One of the biggest challenges in developing an analytical model for fragility analysis is the availability of a bridge database. Based on the available investigation data for the South-North Freeway, a classification is developed and used in the Haz-Taiwan program to evaluate the South-North Freeway bridges. Bridges are classified based on the following structural characteristics:

- Design methodology: Seismic design or conventional design (non-seismic design).

- Number of spans: single span and multiple span bridges.

- Pier type: Single column piers, multiple column bents and pier walls.

- Span continuity: continuous and simply supported.

This classification scheme incorporates various parameters that effect damage into fragility analysis and provides a means to obtain better fragility curves when data become available. A total of 16 classes of bridges for this research is defined this way and shown in Table 1. 
Table 2 Ductility and displacement limits for each damage state

\begin{tabular}{|c|c|c|c|}
\hline \multicolumn{3}{|c|}{$\begin{array}{c}\text { Ductility limits for weak pier and strong } \\
\text { bearings }\end{array}$} & \multirow{2}{*}{$\begin{array}{c}\text { Displacement limit } \\
\text { Weak bearings and } \\
\text { strong pier }\end{array}$} \\
\hline Damage state & Seismic design & $\begin{array}{l}\text { Conventional design } \\
\text { (non-seismic design) }\end{array}$ & \\
\hline Slight & Ductility $R=2.0$ & Ductility $R=1.0$ & Yield displacement \\
\hline Moderate & $R=4.0$ & $R=\min \left(1+\left(R_{f}-1\right) / 2,2.0\right)$ & $10 \mathrm{~cm}$ \\
\hline Extensive & $R=6.0$ & $R=\min \left(R_{f}, 3.0\right)$ & $20 \mathrm{~cm}$ \\
\hline Complete & $R=9.0$ & $\begin{array}{l}R=4.5 \text { or pier reach } \\
\text { its ultimate capacity }\end{array}$ & $\min (40 \mathrm{~cm}, 2 N / 3)$ \\
\hline
\end{tabular}

$R_{f}$ : corresponding ductility at occurrence of flexure to shear failure.

$N$ : seat length of a girder at the support.

\section{DEFINITIONS OF DAMAGE STATES}

To obtain the fragility curve, damage states need to be quantified in terms of spectral displacement or drift. The relationship between the damage state and the displacement is difficult to establish. It requires a large amount of damage data and sound engineering judgment. In this study, based on the methodology adopted in HAZUS, a total of five damage states is defined for highway bridge components. These damage states are none, slight, moderate, extensive and complete damage. Table 2 presents the failure mechanisms and criteria resulting in different damage states adopted in developing analytical curves in this paper.

The detailed description for bridge components of damage states is as follows:

- Slight Damage: Minor cracks and spalling at the column, abutment, girder or deck; cracks at shear key, cracks at expansion joint or approach slab.

- Moderate damage: Columns experiencing moderate cracks and spalling, abutment failure without collapse, shear key failure or restrainer failure without unseating.

- Extensive damage: Any column degrading without collapse or shear failure, significant movement at connections, significant offset of abutment.

- Complete damage: Any column collapse or large movement of connections, deck collapse, tilting of substructure due to ground failure.

\section{DEVELOPMENT OF ANALYTICAL FRAGILITY CURVES}

Two types of bridge fragility curves are used in Haz-Taiwan: one is based on peak ground acceleration (PGA) and the second is based on permanent ground displacement (PGD). In this research the fragility curves are developed as a function of PGA. The current fragility curves for PGD used in HAZUS are adopted with slight modification based on the foundation type for the Haz-Taiwan program.

Researchers, in deriving the fragility curves, generally adopt one of three methods, which are the empirical statistical method, the seismic code method, and the analytic method. The empirical statistical method provides the best estimation for fragility curves when sufficient and reliable post-earthquake damage data are available. However, very few reliable bridge damage data due to ground shaking in Taiwan can be used to conduct the statistical fragility analysis, especially when considering seismic fragility for various bridge classes. The seismic code method, adopted by HAZUS 97 (RMS, 1997), estimates the seismic capacity of certain types of structure based on the local code provisions. This method is very simple to implement, but the results may be highly inaccurate. It may lose relevance to seismic capacity due to ductility details of structural components, and this method is not suitable for the bridge structure because other additional contributors to lateral resistance cannot be incorporated into the capacity analysis. The proposed nonlinear static analysis method for development of analytical fragility curve in terms of PGA is discussed as follows:

\section{Demand Spectrum}

The demand spectrum is given in the form (MOTC, 2001, FEMA, 1997)

$$
S_{a}= \begin{cases}F_{a} S_{D}^{S}\left(0.4+3 T / T_{0}\right) ; & T \leq 0.2 T_{0} \\ F_{a} S_{D}^{S} ; & 0.2 T_{0}<T<T_{0} \\ F_{v} S_{D}^{1} / T ; & T \geq T_{0}\end{cases}
$$

with

$$
T_{0}=\frac{F_{v} S_{D}^{1}}{F_{a} S_{D}^{S}}
$$




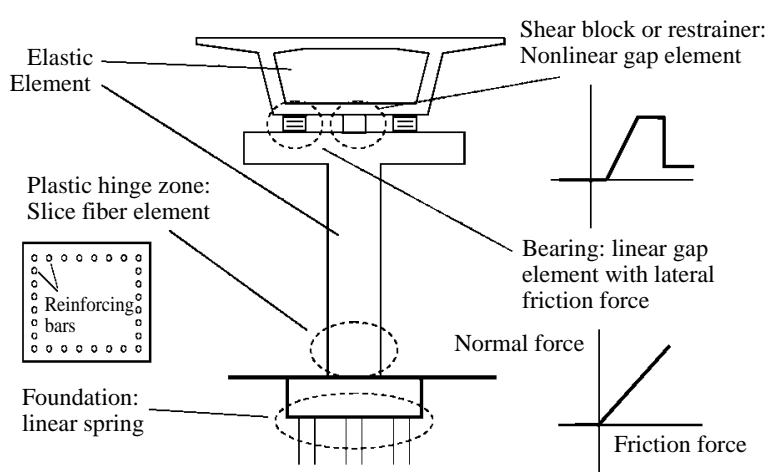

Fig. 2 Modeling for the components usually used in the static push over analysis

where $S_{D}^{S}=S_{a}(T=0.3)$ is the demand spectral acceleration at period $T=0.3 \mathrm{sec}$, the subscript $D$ means demand and superscript $S$ means short period. $S_{D}^{1}=S_{a}$ $(T=1.0)$ is the demand spectral acceleration at $T=1.0$ sec. $S_{D}^{S}$ and $S_{D}^{1}$ are obtained by (1) Specify attributes of the earthquake scenario including location, depth, magnitude, fault rupture type, etc. (2) Determine the ground motion levels for the bedrock using appropriate attenuation laws. $F_{a}$ and $F_{v}$ are the site amplification coefficients for short and long period spectrum, respectively. They are functions of the soil type and the ground shaking level $S_{D}^{S}$ and $S_{D}^{1}$ (MOTC, 2001).

\section{Bridge Capacity Curve}

The first step for developing the bridge capacity curve is to create a computer model of the prototype bridge structure. The computer program DRAIN-3DX (Prakash and Powell, 1993), with some modification, is adopted as the analysis tool in this research. The modeling rules for the elements and components usually used in push over analysis of bridge structures are briefly summarized in Fig. 2.

The capacity is used to demonstrate the seismic capacity of a bridge structure; its ordinate is usually the base shear $V$ and its abscissa is usually the displacement $\Delta$ at a specified point on the structure. Through nonlinear pushover analysis, the relationship between the base shear $V$ and the displacement $\Delta$ at a specified point can be established, and the push over curve can be converted to a capacity spectrum by the following equations (ATC40, 1996):

$$
\begin{aligned}
& S_{a}=\frac{V}{W \alpha_{1}} \\
& S_{d}=\frac{\Delta}{P F_{1} \phi_{1,1}}
\end{aligned}
$$

where $P F_{1}$ is the modal participation factor for the first mode, $\alpha_{1}$ is the modal mass coefficient for the

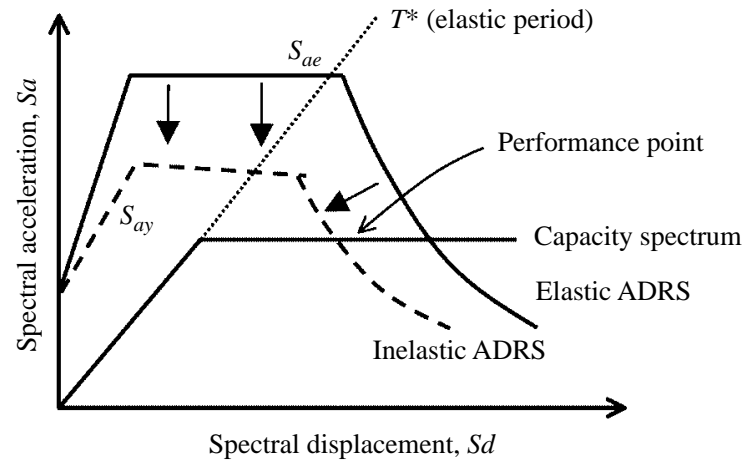

Fig. 3 Performance point and the acceleration-displacement-response-spectrum (ADRS)

first mode, $\phi_{1,1}$ is the modal amplitude at a specified point of the first mode, $W$ is the seismically effective weight of the bridge, $S_{a}$ is the spectral-acceleration and $S_{d}$ is the spectral-displacement.

\section{(i) Bridge Seated on Strong Bearings with Weak Piers}

For a bridge seated on strong bearings with weak piers, in general, the damage will first occur in the pier; For each damage state, determine the ductility demand $R$ and the elastic demand $S_{a e}$ from the target displacement demand $S_{d}$ of selected damage state defined in Table 2 by the following equations (Fig. 3):

$$
\begin{aligned}
& R=S_{d} / S_{d y} \\
& S_{a e}=S_{a y} / F_{u} \\
& F_{u}= \begin{cases}R ; & T \geq T_{0} \\
\sqrt{2 R-1}+(R-\sqrt{2 R-1}) & 0.6 T_{0} \leq T \leq T_{0} \\
\times \frac{T-0.6 T_{0}}{0.4 T_{0}} ; & \\
\sqrt{2 R-1} ; & 0.2 T_{0} \leq T \leq 0.6 T_{0} \\
\sqrt{2 R-1}+(\sqrt{2 R-1}-1) & T_{0} \leq 0.2 T_{0} \\
\times \frac{T-0.2 T_{0}}{0.2 T_{0}} ; & \end{cases}
\end{aligned}
$$

where $S_{d}$ is the spectral-displacement demand and its value depends on the corresponding damage state, $S_{d y}$ and $S_{a y}$ are the yield spectral displacement and yield spectral acceleration of the capacity spectrum, respectively. $F_{u}$ is the structure system reduction factor, Eq. (6) was proposed by MOTC (2001) and Loh et al. (2002), using the Bouc-Wen model (Baber and Noori, 1985; Sues et al., 1988) conducted the 


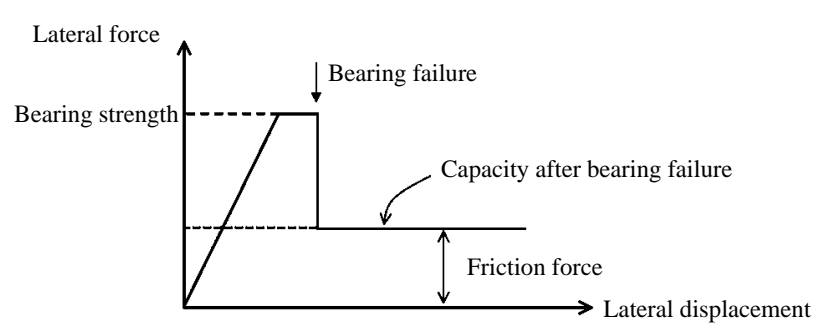

Fig. 4 The relationship between the lateral force and displacement for the bearing

nonlinear dynamic analysis to get the relationships between the ductility and the structure system reduction factor for different hysteretic models, and showed this equation is reliable and suitable for a major hysteretic model. If the transit period $T_{0}$ at Eq. (1) and Eq. (6) is set as $T_{0}=0.4 \mathrm{sec}$ for hard site class (ATC40, 1996), the relationship between the peak ground acceleration and the spectral acceleration is $P G A=$ $0.4 F_{a} S_{D}^{S}$ (and $S_{D}^{1}=0.4 S_{D}^{S}$ ). Then the relationship between peak ground acceleration (PGA) and spectral displacement $S_{d}$ of the bridge structure can be obtained by using the following equation

$$
P G A=\frac{S_{a y} F_{u}}{C}
$$

where $C$ is the normalized demand spectrum defined in Eq. (1), (ie. $P G A \times C=S_{a}$ ).

\section{(ii) Bridge Seated on Weak Bearings with Strong Piers}

For a bridge seated on weak bearings with strong piers, after the failure of bearings, only the friction force between the superstructure and the cap beam are resident to resist the seismic force. Fig. 4 shows the force-displacement relationship for a bridge seated on strong bearings with weak piers. The corresponding damage states for those types of bridges are also shown in Table 2. After bearing failure, the corresponding peak ground acceleration for each damage states is calculated as follows (Basöz and Mander, 1998)

$$
P G A=2 \pi \sqrt{\frac{S_{d}}{S_{a y} g}} S_{a y} B_{L}=2 \pi \sqrt{\frac{S_{d} \mu}{g}} B_{L}
$$

where $S_{d}$ is the demand displacement of damage state, $\mu$ is the friction coefficient and $B_{L}$ is the long period spectrum reduction factor due to damping, which is set to 2.0 in this research.

\section{Evaluation of Fragility Curves}

A fragility curve is a probabilistic measure for the fragility of a model structure under various levels of ground shaking or ground failure. The

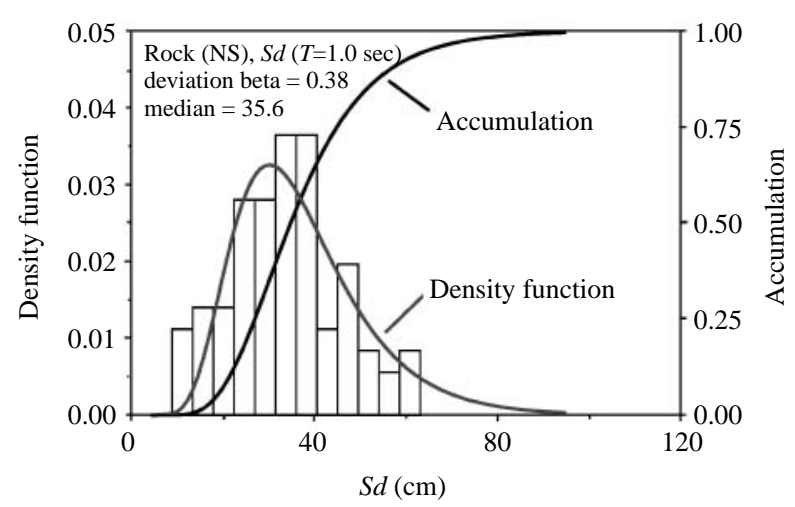

(a)

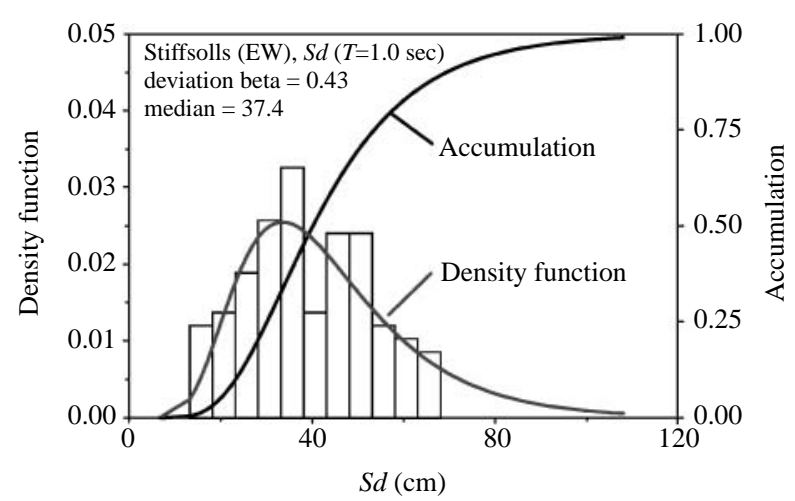

(b)

Fig. 5 Distribution of the density function of demand spectral displacement and its corresponding cumulative distribution for site class (a) type-1 and (b) type-2

fragility curve is defined by a medium value of PESH (Potential Earth Science Hazard) demand parameter and a standard deviation $(\beta)$ of the natural logarithm of the spectral displacement for damage state. Like the HAZUS, the Haz-Taiwan adopts four damage states; slight, moderate, extensive and complete damage to describe the bridge damage state. If the Peak Ground Acceleration value is selected as the demand parameter for ground shaking, for a specified damage state, the elastic demand spectral-acceleration can be determined by the procedure shown in the foregoing, and through Eq. (7) or Eq. (8) the corresponding PGA value can be found by using the relationship $\mathrm{PGA}=0.4 S_{D}^{S}$ to represent the medium value of the fragility curve.

In general, the total variability of the damage state, $\beta$, is constituted by the uncertainty of the damage state threshold, the variability of the bridge capacity and the uncertainty of ground motion demand. In this research, only the uncertainties of ground motion demand are used to represent the total variability of the damage state, and the standard deviation of the natural logarithm of the spectral displacement from the recorded earthquake data is adopted as the reference for representing the $\beta$ value. Fig. 5 shows the distribution of the spectral displacement 
and its corresponding cumulative distribution, which is analyzed from the earthquake data for site class type- 1 and -2 classified by Taiwan seismic design code (MOTC, 2001). From these figures, the standard deviation of the spectral displacement is around 0.4 , this is similar to the value adopted in HAZUS. Then this value is taken into account as the base reference for the standard deviation of the fragility curve.

In this research, for each bridge class there are more than 3 bridges selected as prototype structures in the analytical fragility analysis. The medium value of the fragility curve for each bridge class is based on the mean value of the analyzed results of those prototype bridges and practical engineering judgment. Some specific examples of analytical curves are presented in the following sub-sections.

\section{(i) Example 1: Continuous - Multi Column - Conven- tional Design (Class Type 6C)}

One of the selected prototype bridges for the class type $6 \mathrm{C}$ is a highway bridge, located at the Keelung-Neihu section. The superstructure of this bridge is assembled from 6 PCI-girders and concrete slabs, the substructure is multiple columns bent with pile foundation. The configuration of this bridge is shown in Fig. 6a, and the analytical model is shown in Fig. 6b. Fig. 6c shows the capacity curve of this bridge and the corresponding ground shaking level (PGA) in the longitudinal direction, the description of each damage state is also shown in the figure. In this example, because the seismic capacity is dominated by the longitudinal direction, only the analysis results in the longitudinal direction have been presented. Fig. 6d displays the fragility curves of each damage state of this bridge. In this Figure, the medium value (50\% exceeding probability) represented by PGA for the slight, moderate, extensive and complete damages are 0.2, 0.62, 0.90 and $1.06 \mathrm{~g}$, respectively. As shown in Fig. 6c, when the spectral displacement reaches about $3.5 \mathrm{~cm}$, the approach slab or expansion joints may have some damage, also these type damages will not affect the safety of the bridge but the function of the bridge will be affected slightly, so define the bridge at this stage as having slight damage. The moderate damage state is corresponding to the state where the back wall of the abutment loss its strength. The extensive and complete damage states are mainly dominated by the shear strength and ductile demand of piers.

\section{(ii)Example 2: Simply Supported - Pier Wall - Con- ventional Design (Class Type 4C)}

One of the selected prototype bridges of the class

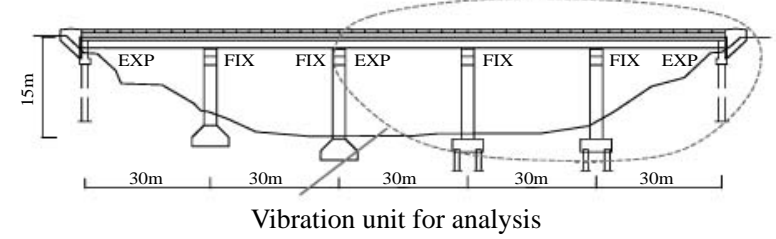

(a)

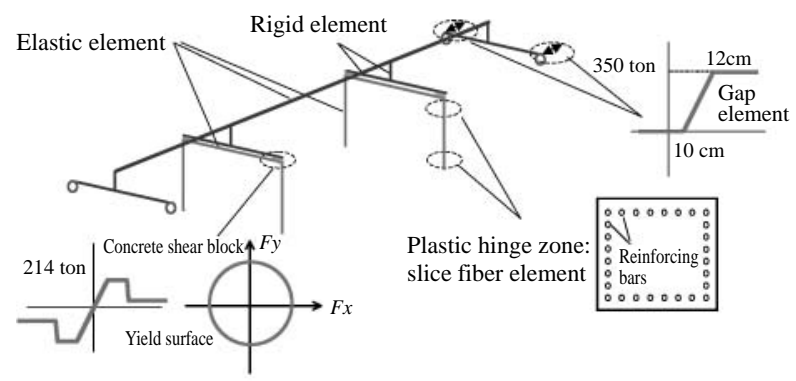

(b)

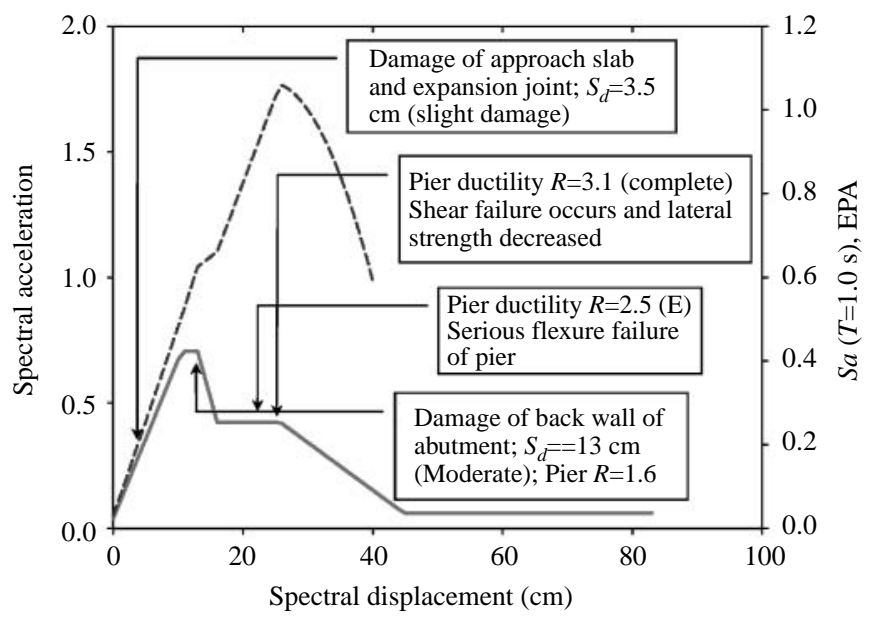

(c)

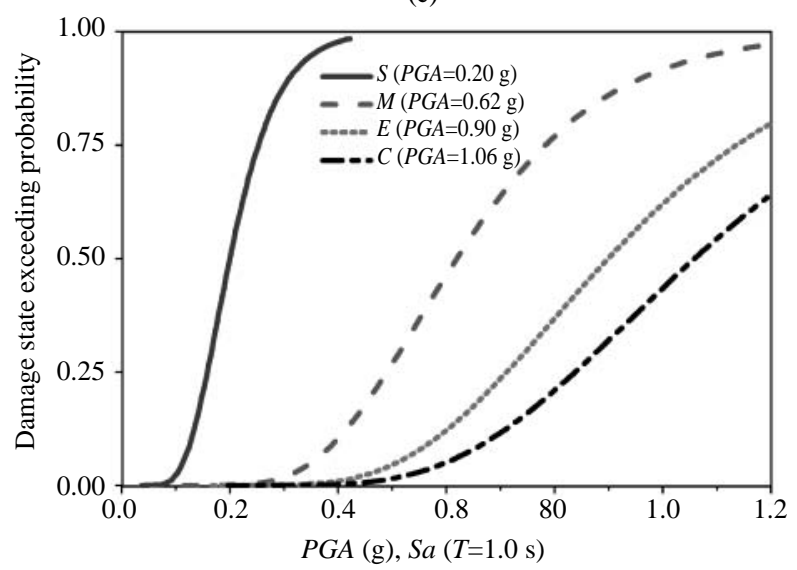

(d)

Fig. 6 (a) Configuration of the analyzed bridge; Freeway Keelung-Neihu Section; (b) the analytical model of the analyzed brdige; (c) the analytical capacity curve (solid line, scale shown on left-hand side ordinate) and the ground shaking level (dash line, scale shown on right-hand side ordinate) of the bridge; (d) the analytical fragility curves of the brdige for each damage state 
type $4 \mathrm{C}$ is the freeway bridge located in the ShinJuMiaoLi section. The superstructure of this bridge is assembled from 8 PCI-girders and concrete slabs, the substructure is multiple columns bent with shallow foot foundation. The profile, pier type, bearing and abutment of this bridge are shown in Fig. 7a. Fig. 7b shows the capacity curve of this bridge and the corresponding description of each damage state is also shown in this figure.

\section{(iii) Structural Characteristics of South-North Free- way}

The age of most of the bridges on the SouthNorth freeway is about 25 years, some broader lanes were constructed about 8-10 years ago. After the review of the available bridge database for the SouthNorth freeway, the structural characteristics of those bridges are found and stated as follows:

- The PCI girder is used in the superstructure and the span length is about $25 \mathrm{~m}-40 \mathrm{~m}$ for most bridges. For multiple span-simply supported bridges, live load continuous deck is adopted.

- Seat length of bridges is about $70-80 \mathrm{~cm}$; it can comply with the seismic design code in Taiwan.

- For the conventionally designed bridges, the volume ratio of lateral restraining reinforcement is about $15-25 \%$ of code requirement for pier wall and $30-70 \%$ for column.

- The rubber bearing is adopted for those bridges with PCI girder as superstructure; steel pin with rubber bearing used as the fixed bearing; concrete seismic block is used as the unseating prevention device.

- For the pier wall, the shear strength of the pier is generally greater than the shear strength of the seismic block. Then the seismic capacity of the pier wall bridge is dominated by the strength of the seismic block.

- For a column pier, the strength of the pier is generally less than the shear strength of the seismic block. Then the seismic capacity for the transverse direction of the column pier bridge is controlled by the strength of the pier.

The damage states of slight and moderate damage are generally controlled by the strength of the abutment or bearing or seismic block, but the types and strength of abutment and seismic block possess a large degree of uncertainty.

In this research, the analytical fragility for the selected prototype bridges is analyzed, and then determination of the medium value of the fragility curve for each bridge class is based on the mean value of the analyzed results and practical engineering judgment. The structural characteristics of SouthNorth freeway bridges mentioned above are helpful

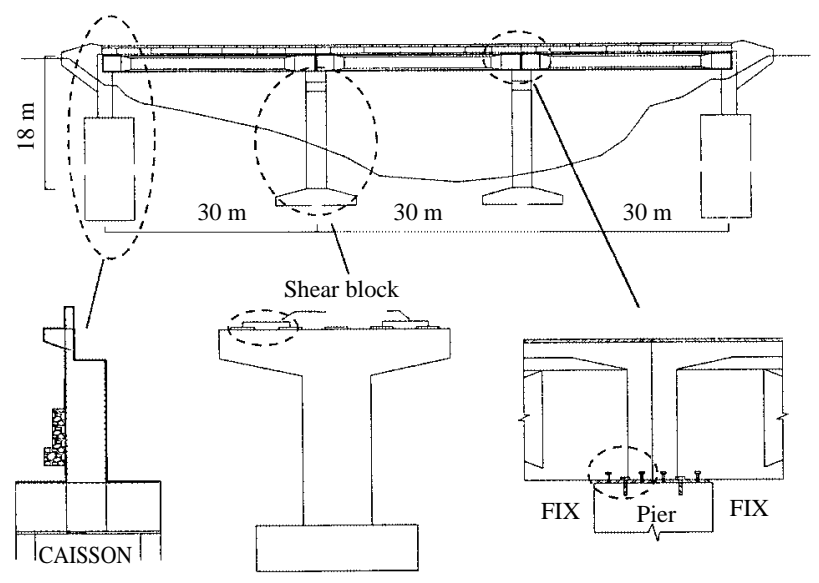

(a)

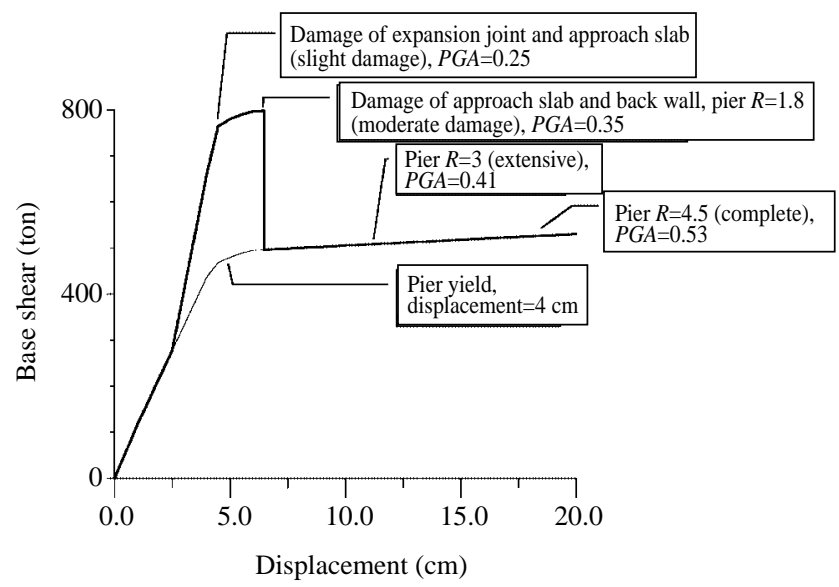

(b)

Fig. 7 (a) The profile, pier type, bearing and abutment of the selected Type 4C bridge; (b) the analytical capacity curve and damage states of the bridge

to modify the medium value and standard deviation of the fragility curve. Table 3 shows the damage functions (medium value and standard deviation) for each bridge class due to ground shaking and ground failure. The damage functions due to ground failure were not obtained from this research, but adopted those values used in HAZUS99.

\section{CONCLUDING REMARKS}

The extensive vulnerability of the existing bridge inventory, as revealed by this earthquake, must be addressed before other equally destructive earthquakes strike again in Taiwan. The seismic risk assessment methodology for bridge structures has been developed. In this method, the fragility curves of different type of bridge were developed from this study. The result can be applied to seismic loss estimation for the transportation system. Computation clarity and the data obtained are limited, and some assumptions have been made in this study. To improve the modeling of 
Table 3 Fragility of bridges

\begin{tabular}{ccccccccc}
\hline & \multicolumn{3}{c}{$\begin{array}{l}\text { PGA }(\mathrm{g}) \text { for damage functions due } \\
\text { to ground shaking }\end{array}$} & \multicolumn{5}{l}{$\begin{array}{l}\text { PGD }(\mathrm{cm}) \text { for damage functions } \\
\text { due to ground failure }\end{array}$} \\
\cline { 2 - 8 } Class & Slight & Moderate & Extensive & Complete & Slight & Moderate & Extensive & Complete \\
\hline TYPE 1C & 0.30 & 0.60 & 0.98 & 1.39 & 10.0 & 10.0 & 15.0 & 45.0 \\
TYPE 1S & 0.30 & 0.60 & 0.98 & 1.39 & 30.0 & 30.0 & 45.0 & 135.0 \\
TYPE 2C & 0.20 & 0.28 & 0.46 & 0.58 & 10.0 & 10.0 & 15.0 & 35.0 \\
TYPE 2S & 0.22 & 0.35 & 0.65 & 0.83 & 30.0 & 30.0 & 45.0 & 105.0 \\
TYPE 3C & 0.20 & 0.30 & 0.52 & 0.66 & 10.0 & 10.0 & 15.0 & 35.0 \\
TYPE 3S & 0.22 & 0.40 & 0.72 & 0.90 & 30.0 & 30.0 & 45.0 & 105.0 \\
TYPE 4C & 0.20 & 0.30 & 0.38 & 0.50 & 10.0 & 10.0 & 15.0 & 35.0 \\
TYPE 4S & 0.22 & 0.35 & 0.55 & 0.70 & 30.0 & 30.0 & 45.0 & 105.0 \\
TYPE 5C & 0.20 & 0.28 & 0.40 & 0.50 & 10.0 & 10.0 & 15.0 & 25.0 \\
TYPE 5S & 0.22 & 0.40 & 0.60 & 0.80 & 30.0 & 30.0 & 45.0 & 75.0 \\
TYPE 6C & 0.23 & 0.38 & 0.53 & 0.70 & 10.0 & 10.0 & 15.0 & 25.0 \\
TYPE 6S & 0.26 & 0.42 & 0.75 & 0.95 & 30.0 & 30.0 & 45.0 & 75.0 \\
TYPE 7C & 0.23 & 0.32 & 0.40 & 0.50 & 10.0 & 10.0 & 15.0 & 25.0 \\
TYPE 7S & 0.25 & 0.35 & 0.57 & 0.70 & 30.0 & 30.0 & 45.0 & 75.0 \\
TYPE 8C & 0.22 & 0.35 & 0.52 & 0.69 & 10.0 & 10.0 & 15.0 & 25.0 \\
TYPE 8S & 0.24 & 0.41 & 0.69 & 0.90 & 30.0 & 30.0 & 45.0 & 75.0 \\
\hline Deviation $\beta$ & 0.50 & 0.45 & 0.40 & 0.40 & 0.20 & 0.20 & 0.20 & 0.20 \\
\hline
\end{tabular}

simulations for foundation behavior, nonlinear soilstructure interaction and additional contributors to lateral resistance, further studies and statistical analyses need to be done. Furthermore, the bridge class must be extended to be able to accommodate other highway bridges in Taiwan, and the analytical fragility curves need to be examined by comparing with empirical fragility curves when the database is available.

\section{ACKNOWLEDGMENTS}

The authors gratefully acknowledge the financial support granted by the T. Y. Lin Consultants, Taiwan, and the National Science Council (92-2211E-390-002), R.O.C. The facilities for research provided by National Center for Research on Earthquake Engineering are also highly appreciated.

\section{NOMENCLATURE}

$B_{L} \quad$ long period spectrum reduction factor due to damping

$F_{a} \quad$ site amplification coefficients for short period spectrum

$F_{v} \quad$ site amplification coefficients for long period spectrum

$F_{u} \quad$ structure system reduction factor

$P F_{1} \quad$ modal participation factor for the first mode

$R \quad$ ductility demand

$S_{a} \quad$ spectral acceleration

$S_{d} \quad$ spectral displacement
$S_{D}^{1} \quad$ demand spectral acceleration at period $T=1.0$ $\sec$

$S_{D}^{S} \quad$ demand spectral acceleration at period $T=0.3$ sec

$S_{a y} \quad$ yield spectral acceleration of the capacity spectrum

$S_{d y} \quad$ yield spectral displacement of the capacity spectrum

$W \quad$ seismically effective weight of the bridge

$\alpha_{1} \quad$ modal mass coefficient for the first mode

$\beta \quad$ standard deviation of the spectral displacement for damage state

$\phi_{1,1}$ modal amplitude at specified point of the first mode

$\mu \quad$ friction coefficient and

\section{REFERENCES}

Applied Technology Council (ATC-40), 1996, "Seismic Evaluation and Retrofit of Concrete Buildings," California Seismic Safety Commission, CA, USA.

Baber, T. T., and Noori, M. N., 1985, "Random Vibration of Degrading, Pinching Systems," Journal of Engineering Mechanics, ASCE, Vol. 111, No. 8, pp. 1010-1026.

Basöz, N., and Mander, J., 1998, "Enhancement of the Highway Transportation Lifeline Module in HAZUS (Draft)," National Institute of Building Sciences, Washington, D.C., USA.

Federal Emergency Management Agency (FEMA), 
1997, NEHRP Guidelines for the Seismic Rehabilitation of Buildings, Washington, D.C., USA.

Loh, C. H., Liao, W. I., Chai, J. F., Jean, W. Y., and Teng, T. J., 2002, Study on Performance Objective and Relative Technologies for the Seismic Design of Building Structures, Architecture and Building Research Institute, Ministry of Interior Affair, Taiwan (in Chinese).

Ministry of Transportation and Communication (MOTC), 2001, The Modification of Seismic Design Provision and Commentary for Highway Bridges (Drafts), Taiwan (in Chinese).

Prakash, V., and Powell, G. H., 1993, DRAIN-3DX: Base Program User Guide, Version 1.10, Department of Civil Engineering, University of California, Berkeley, CA, USA.

Risk Management Solutions, Inc., 1997, Earthquake Loss Estimation Methodology - HAZUS97 Technical Manual, Federal Emergency Management
Agency, Washington, D.C., USA.

Risk Management Solutions, Inc., 1999, Earthquake Loss Estimation Methodology - HAZUS99 Technical Manual, Federal Emergency Management Agency, Washington, D. C., USA.

Sues, R. H., Mau, S. T., and Wen, Y. K., 1988, "System Identification of Degrading Hysteretic Restoring Force," Journal of Engineering Mechanics, ASCE, Vol. 1145, No. 5, pp. 833-846.

Yeh, C. H., Lee, C. Y., and Loh, C. H., 1999, "Framework of Earthquake Loss Estimation Method in Taiwan," Proceedings 1999 Workshop on Disaster Prevention/Management and Green Technology, Foster City, CA, USA.

Manuscript Received: Sep. 16, 2002

Revision Received: Oct. 02, 2003 and Accepted: Nov. 21, 2003 